\section{Chronic pain: a practical approach to multimodal management - diagnosis and surgical options for chronic joint pain}

\section{Kevin Parsons}

The successful management of chronic joint pain is one of the most common problems faced in practice. Effective management relies initially on reaching the correct diagnosis and a thorough consideration of the multitude of treatment options available. Evidence supporting the different treatment options should also be carefully considered.

The most common cause of joint pain is osteoarthritis and it has a reported prevalence of $20 \%$ in adult dogs. It is important to remember that primary osteoarthritis (i.e. osteoarthritis without an underlying/initiating aetiology) is uncommon. Underlying causes of chronic joint pain leading to osteoarthritis include historic fractures, elbow dysplasia, hip dysplasia, osteoarthritis, immunemediated polyarthritis, joint infection and neoplasia.

Accurate identification of the cause of chronic joint pain requires a thorough physical and orthopaedic examination. Once the source of discomfort is localized to a joint, appropriate diagnostic tests should be selected to obtain a diagnosis. It is always important to avoid unnecessary costs and therefore choose only appropriate tests. Orthogonal radiographs are usually indicated, however in some cases computed tomography (CT) may be beneficial. It is important to emphasize that the interpretation of radiographic and $\mathrm{CT}$ findings should be based on the physical examination findings. In other cases, although much less commonly, magnetic resonance imaging and ultrasonography can be useful. Following imaging, arthrocentesis may be required. Further diagnostic tests that can be worthwhile include arthroscopy.

The management of chronic joint pain depends on the diagnosis. If an underlying condition is identified treatment should be aimed at that (e.g. polyarthritis, septic arthritis, cruciate disease, neoplasia). The treatment of osteoarthritis is more generic.

Numerous strategies have been described for the management of osteoarthritis ranging from lifestyle changes, exercise modification, weight loss, non-steroidal anti-inflammatory drugs, physiotherapy, hydrotherapy, acupuncture, nutraceuticals, polysulfated glycosaminoglycan, omega-3 fatty acids, corticosteroids, amantadine and surgery.
There are many cases where conservative treatment is appropriate. Referral for conservative treatment is an option. If embarking on conservative treatment, all patients should be evaluated regularly to assess their progress. Patients should be evaluated at regular intervals because some signs of chronic pain appear not to be recognized by some owners.

Surgical treatments are available for the treatment of chronic joint pain and some of these are well proven and may be more appropriate than conservative treatment. The specific surgical options available for management of chronic joint pain are influenced by the joint involved. In general, surgical options available include:

\section{Arthrodesis \\ Total joint replacement \\ Excision arthroplasty \\ Osteotomy techniques (elbow dysplasia) \\ - Joint debridement and micropick surgery.}

When chosen appropriately and well executed, surgery can offer a great solution to the management of some causes of chronic joint pain.

\section{KEY LEARNING OBJECTIVES}

- Be able to diagnose chronic joint pain through the application of appropriate diagnostic tests and identify if an initiating cause is present

- Have an improved understanding of when surgery is appropriate for the management of chronic joint pain

- Have knowledge of surgical options for common causes of chronic joint pain

\section{MULTIPLE CHOICE QUESTIONS}

1. Which of the following is the most uncommon initiating cause of chronic joint pain in the dog?
(A) Primary osteoarthritis
(B) Joint trauma
(C) Immune-mediated polyarthritis
(D) Congenital conditions such as hip dysplasia

2. Which of the following is a contraindication to total hip replacement surgery?
(A) Small patient size $(<6 \mathrm{~kg})$
(B) Concurrent neurological disease
(C) A positive clinical response to
(D) Patient age

3. When considering chronic pain associated with radial-carpal joint hyperextension, which of the following is the most appropriate initial treatment option?
(A) Cage rest and anti-inflammatory medication
(B) Splinting with a customized splint
(C) Pancarpal arthrodesis
(D) Partial carpal arthrodesis 